\title{
Construção empírica e validação de um instrumento de avaliação global de saúde mental ${ }^{*}$
}

\section{Empirical construction and validation of an overall evaluation of mental health}

\author{
Geilsa Soraia Cavalcanti Valente ${ }^{1} \cdot$ Elaine Antunes Cortez $^{2} \bullet$ Carlos Alberto da Cruz Sequeira $^{3}$
}

\begin{abstract}
RESUMO
Com a realização deste estudo, pretendeu-se elaborar e validar o construto e a confiabilidade de uma escala de avaliação global do nível de saúde mental. A escala foi construída a partir da busca on-line e localização de 63 modelos de avaliação em saúde mental e posteriormente aplicada em 266 pessoas dos diversos grupos humanos. 0 processo envolveu a validação do conteúdo por 25 especialistas, participantes do Grupo Internacional de Investigação em Enfermagem de Saúde mental, testes piloto, medição da confiabilidade utilizando o coeficiente alfa de Cronbach e análise fatorial. Coeficientes de Cronbach adequados foram obtidos nas três dimensões e a análise fatorial confirmou as dimensões da escala. Conclui-se que pode-se inferir que a escala de avaliação global do nível de saúde mental é um instrumento confiável e válido. Os resultados revelam que os itens apresentam boas características psicométricas na amostra, podendo constituir um instrumento útil na avaliação da saúde mental nos diversos grupos humanos.
\end{abstract}

Palavras-chave: Saúde mental; Psicometría; Avaliação de Programas e Instrumentos de Pesquisa.

\begin{abstract}
In this study, it was intended to develop and validate the construct and the reliability of a global rating scale of mental health level. The scale was constructed from the online search and location of 63 evaluation models in mental health and later applied to 266 people of various human groups. The process involved the validation of content by 25 experts, participants of the International Research Group on Mental Health Nursing, pilot testing, reliability of measurement using the Cronbach's alpha coefficient and factor analysis. Adequate Cronbach coefficients were obtained in three dimensions and the factor analysis confirmed the dimensions of scale. It concludes that it can be inferred that the overall assessment of the scale of mental health level is a reliable and valid instrument. The results show that the items have good psychometric characteristics in the sample and can be a useful tool in the assessment of mental health in different human groups.
\end{abstract}

Keywords: Mental Health; Psychometrics; Evaluation Program and Research Instruments.

\footnotetext{
1. Geilsa Soraia Cavalcanti Valente. Pós-Doutora em Enfermagem. Professora do Departamento de Fundamentos de Enfermagem e Administração da Escola de Enfermagem Aurora de Afonso Costa /Universidade Federal Fluminense. Niterói, RJ. Brasil. Bolsista CNPq do Programa Ciência Sem Fronteiras para o pós-doutoramento na Escola Superior de Enfermagem do Porto - ESEP. Portugal.Email: geilsavalente@yahoo.com.br

2. Elaine Antunes Cortez. Doutora em Enfermagem. Professora do Departamento de Enfermagem em Saúde da Mulher e Psiquiatria da Escola de Enfermagem Aurora de Afonso Costa /Universidade Federal Fluminense. Niterói, RJ. Brasil. Bolsista CNPq do Programa Ciência Sem Fronteiras para o pós-doutoramento na Escola Superior de Enfermagem do Porto - ESEP. Portugal. Email: nanicortez@hotmail.com

3. Carlos Alberto da Cruz Sequeira. Doutor em Enfermagem. Professor coordenador da Escola Superior de Enfermagem do Porto. Portugal. Coordenador do Grupo de Investigação - NurID: Inovação e Desenvolvimento em Enfermagem - CINTESIS - Center for health technology and services research - Faculdade de Medicina da Universidade do Porto - FMUP. Presidente da Sociedade Portuguesa de Enfermagem de Saúde Mental. Email: carlossequeira@esenf.pt

*Pesquisa desenvolvida como parte dos trabalhos do pós-doutoramento realizado na Escola Superior de Enfermagem do Porto - Portugal. $2014 / 2015$.
} 


\section{INTRODUÇÃO}

A saúde é o bem mais precioso que as pessoas tentam preservar ao longo do seu ciclo de vida e é, também, um fenômeno com forte componente social, psicológico, mental, espiritual e físico. Numa perspectiva transcultural, é quase impossível definir Saúde Mental (SM) de uma forma completa. Concorda- se quanto ao fato de que essa é algo mais do que a ausência de distúrbios mentais. É importante compreender a SM e, de modo mais geral, o funcionamento mental, porque daí advém a base sobre a qual se formará uma compreensão mais completa do desenvolvimento dos distúrbios mentais e comportamentais. ${ }^{1}$

A Saúde Mental é determinada pela inter-relação de fatores físicos, ambientais, sociais, psicológicos e espirituais, e é parte integrante de todo o desenvolvimento humano. Estes fatores podem se apresentar de forma positiva (bem-estar psicológico) ou de forma negativa (distress psicológico) quando não se verifica uma adequada adaptação. $^{2}$

A maioria das abordagens da saúde mental, sob o ponto de vista da investigação, tem privilegiado o estudo de psicopatologia, com base na existência de sinais e sintomas, em vez do estudo das qualidades que podem proteger as pessoas com problemas de SM. ${ }^{1} \mathrm{~A}$ avaliação dos seus aspectos positivos constitui-se como um recurso determinante para a promoção da saúde das pessoas, privilegiando-se um modelo de Saúde Mental Positiva, em detrimento de um modelo centrado na doença. ${ }^{3}$

A Saúde Mental Positiva pode ser definida como um valor em si (sentir-se bem) ou como uma capacidade para perceber, compreender e interpretar o meio para se adaptar e alterá-lo, se necessário, para pensar e se comunicar com os outros. ${ }^{4}$ Os investigadores da área da SM têm manifestado muito interesse na forma como a saúde pode ser influenciada pelas dimensões positivas das experiências (integração social, autoeficácia, controle percebido entre outros).

Nos últimos anos, os conceitos da Saúde Mental Positiva (efeito positivo, bem-estar, benefício) têm tido uma valorização crescente na investigação em saúde. Numa perspectiva salutogênica, as variáveis promotoras de Saúde Mental assumem uma importância acrescida, na qual é possível entendê-la como indicador de integração e adaptação. ${ }^{2}$

Diversos autores procuraram compreender a resposta do organismo perante as agressões do exterior, numa perspectiva biológica do stress, mas são múltiplas as diferentes perspectivas e Modelos compreensivos e explicativos do stress. A visão do stress enquanto estímulo é sensível a uma taxonomia de estressores ambientais que se podem definir na duração ou cronicidade, das diferenças individuais face à vulnerabilidade dos estressores e no tipo de respostas ao estímulo numa interação estímulo-resposta.

Assim, entende-se a importância de elaborar um instrumento que avalie não só o lado negativo, que corresponde aos fatores de risco para a SM, mas um instrumento que também possa medir os fatores protetores dos indivíduos, o lado positivo, tendo em vista que a vida é um processo dinâmico em que hora o indivíduo encontrase mais propenso ao risco, hora encontra-se mais protegido deste.

Vale salientar que o termo psicossocial tem sido utilizado para referir uma grande variedade de fatores psicológicos e sociais que se relacionam com a saúde e a doença mental. ${ }^{3}$ Neste sentido, considera-se, a partir da coexistência entre 0 individuo e o ambiente, que existem os fatores de risco para o estresse, que decorrente da vulnerabilidade mental do indivíduo, pode ocasionar a crise e desencadear doença mental; e os fatores protetores, que em decorrência da sua saúde mental, levam o indivíduo ao equilíbrio emocional, resultando na saúde mental positiva.

A necessidade deste estudo surgiu pela constatação da inexistência de instrumentos validados que avaliem o estado geral de saúde mental dos diversos grupos humanos. Assim, tem como objetivo validar o construto e a confiabilidade de uma escala de avaliação global do nível de saúde mental.

\section{MÉTODOS}

\section{Procedimentos}

$\mathrm{Na}$ construção do instrumento de Avaliação Global do Nível de Saúde Mental - AGNSM - incluíram-se duas fases: uma de desenvolvimento de itens, a partir de uma revisão de literatura, onde foram selecionados 26 instrumentos psicométricos disponíveis online contidos em 119 produções científicas, localizadas nas bases de dados: Medical Literature Analysis and Retrieval System Online - MEDLINE, PUBMED e Literatura Latino-Americana e do Caribe em Ciências da Saúde - LILACS, e outra para o teste empírico da fidelidade e validade de instrumentos.

$\mathrm{Na}$ fase inicial, procedeu-se a um trabalho de natureza exploratória, para a construção dos itens do inventário,entre os meses de março a agosto de 2014. Todas as questões foram colocadas numa base Excel, sendo eliminadas as questões repetidas ou com significado similar, até chegar à versão preliminar do instrumento. $\mathrm{Na}$ segunda fase, tornou- se prioritário conhecer a opinião dos peritos, especialistas em saúde mental e participantes do Grupo Internacional de Investigação em Enfermagem de Saúde Mental - GIISAME, composto por pesquisadores do Brasil, Espanha e Portugal, tendo-se utilizado um estudo Delphi para consenso entre os peritos, quando se considerou os itens com uma concordância superior a $70 \%$.

Assim, pretendeu-se obter informação e conhecer a perspectiva destes pesquisadores sobre quais eram os fatores mais importantes, dentre os itens selecionados, a partir de três tipos de grupos de fatores: positivos, negativos e ambíguos, a partir daqui foram agrupadas 
as questões com maior sentido de coerência e criada a versão preliminar do instrumento, resultando em 63 itens, incluindo os de identificação da população.

A seguir, foram convertidos numa escala do tipo Likert, que após avaliação pelos peritos, as investigadoras responsáveis procederam à cotação dos itens do AGNSM. Foi realizado um pré-teste do instrumento organizado na ferramenta Google-drive, com 64 respondentes, quando se observou que as questões do instrumento eram viáveis quanto à coerência. Posteriormente, foi enviado por e mail para diversos contatos do Brasil, Espanha e Portugal, obtendo-se um retorno de preenchimento por 266 pessoas, compondo um quantitativo suficiente para amostragem. 0 tratamento estatístico dos dados teve suporte informático no programa Statistical Package for Social Sciences (SPSS), versão 22.0 para Windows.

Procedeu-se o estudo das características psicométricas do instrumento em termos da análise de fidelidade (consistência interna) e da validade de constructo. Considerando a importância de avaliar a sensibilidade e fidelidade dos resultados, garantindo "o grau de uniformidade e de coerência existente entre as respostas dos sujeitos a cada um dos itens que compõem a prova”. 0 trabalho de campo nesta fase decorreu entre os meses de outubro de 2014 e março de 2015. Quanto aos aspectos éticos, o projeto de pesquisa foi submetido e aprovado no Comitê de ética em pesquisa da Faculdade de Medicina da Universidade Federal Fluminense, Niterói/RJ. Brasil, sob $\mathrm{n}^{\circ}$ CAAE 33733014.9.0000.5243.

\section{Análise Estatística}

Para responder aos objetivos do estudo foram utilizadas, além de técnicas básicas de análise exploratória de dados, como frequência absoluta e relativa, outras três técnicas de análise estatística: a Análise Fatorial, o Alpha de Cronbach e o Coeficiente de Correlação de Spearman. A Análise Fatorial foi utilizada para investigar quais são os fatores latentes do instrumento, com o objetivo de validar o constructo proposto. Já o Alpha de Cronbach foi utilizado para avaliar se o instrumento é confiável e capaz de produzir mensurações estáveis e consistentes. Por fim, o Coeficiente de Correlação de Spearman foi utilizado para avaliar, estatisticamente, as correlações entre as questões abordadas. Todos os testes de hipóteses desenvolvidos nesse trabalho consideraram uma significância de $5 \%$, isto é, a hipótese nula foi rejeitada quando $p$-valor foi menor ou igual a 0,05 .

\section{RESULTADOS}

A análise iniciou-se com uma descrição da amostra em estudo, onde observou-se que: $33 \%$ dos indivíduos possuem até 30 anos, $32 \%$ são graduados/licenciados, $45 \%$ são casados, $50 \%$ possuem renda de 1 a 5 salários mínimos, $43 \%$ é religioso praticamente, $89 \%$ nunca foram vítimas de abuso/violência/negligência e $65 \%$ se consideram felizes, conforme a tabela 1.

Tabela 1: Características individuais dos participantes da pesquisa

\begin{tabular}{|c|c|c|c|}
\hline Variável & Categoria & $\mathbf{N}^{*}$ & $\%$ \\
\hline \multirow{5}{*}{ Faixa etária } & Até 30 anos & 75 & 33 \\
\hline & 31 a 40 anos & 67 & 30 \\
\hline & 41 a 50 anos & 42 & 19 \\
\hline & 51 a 60 anos & 34 & 15 \\
\hline & 61 anos ou mais & 8 & 4 \\
\hline \multirow{5}{*}{ Formação } & Doutor & 27 & 12 \\
\hline & Especialista & 66 & 29 \\
\hline & Graduado/Licenciado & 72 & 32 \\
\hline & Mestre & 52 & 23 \\
\hline & Pós-Doutor & 9 & 4 \\
\hline \multirow{5}{*}{ Estado civil } & Casado(a) & 101 & 45 \\
\hline & Divorciado(a) & 21 & 9 \\
\hline & Mora com companheiro(a) & 14 & 6 \\
\hline & Solteiro(a) & 88 & 39 \\
\hline & Viúvo(a) & 2 & 1 \\
\hline \multirow{5}{*}{ Rendimento } & Nenhum & 22 & 10 \\
\hline & Menos de 1 salário mínimo & 6 & 3 \\
\hline & De 1 a 5 salários mínimos & 113 & 50 \\
\hline & De 6 a 10 salários mínimos & 54 & 24 \\
\hline & Mais de 10 salários mínimos & 31 & 14 \\
\hline \multirow{5}{*}{ Religião } & Não sei me classificar & 9 & 4 \\
\hline & Não, e não acredito em Deus & 10 & 4 \\
\hline & Não, mas acredito em Deus & 27 & 12 \\
\hline & Sim, não praticante & 82 & 36 \\
\hline & Sim, praticante & 98 & 43 \\
\hline \multirow{3}{*}{$\begin{array}{l}\text { Estado } \\
\text { marital }\end{array}$} & Já fui vitima, mas agora não o sou & 22 & 10 \\
\hline & Nunca fui vitima & 201 & 89 \\
\hline & Não sei especificar & 3 & 1 \\
\hline \multirow{5}{*}{$\begin{array}{l}\text { Estado } \\
\text { parental }\end{array}$} & Considero-me feliz & 146 & 65 \\
\hline & Considero-me infeliz & 1 & 0 \\
\hline & Considero-me insatisfeito & 16 & 7 \\
\hline & Considero-me satisfeito & 57 & 25 \\
\hline & Não sei especificar & 6 & 3 \\
\hline \multicolumn{2}{|l|}{ Total } & 266 & 100 \\
\hline
\end{tabular}

Fonte: dados da pesquisa dos autores.

Em relação ao histórico familiar de saúde mental, de acordo com a tabela 2, 45\% não apresentaram histórico, $69 \%$ nunca fizeram uso de medicação controlada, 52\% estão em empregos públicos, $46 \%$ realizaram atividades voluntárias poucas vezes, $38 \%$ utilizaram drogas algumas vezes, $78 \%$ possuem moradias próprias e residem com 
familiares, 53\% possuem uma alimentação saudável (exceto algumas vezes), 52\% afirmam ter tempo e dinheiro para lazer, $35 \%$ realizam atividades físicas regularmente e $62 \%$ têm poucos, mas verdadeiros amigos.

Tabela 2: Características individuais e clínica dos participantes da pesquisa

\begin{tabular}{|c|c|c|c|}
\hline Variável & Categoria & Fi & $\%$ \\
\hline \multirow{5}{*}{$\begin{array}{l}\text { Histórico } \\
\text { familiar }\end{array}$} & Ninguém na família & 102 & 45 \\
\hline & $\begin{array}{l}\text { Pessoas distantes e próximas na } \\
\text { família }\end{array}$ & 25 & 11 \\
\hline & $\begin{array}{l}\text { Pessoas distantes e próximas na } \\
\text { familia, inclusive eu }\end{array}$ & 9 & 4 \\
\hline & Sei de alguém distante na família & 29 & 13 \\
\hline & $\begin{array}{l}\text { Tenho uma pessoa próxima na } \\
\text { família }\end{array}$ & 61 & 27 \\
\hline \multirow{5}{*}{$\begin{array}{l}\text { Medicamento } \\
\text { controlado }\end{array}$} & Faço atualmente & 17 & 8 \\
\hline & Fiz durante um período curto & 27 & 12 \\
\hline & Fiz durante um período longo & 10 & 4 \\
\hline & Fiz uma vez & 16 & 7 \\
\hline & Nunca fiz & 156 & 69 \\
\hline \multirow{5}{*}{$\begin{array}{l}\text { Situação } \\
\text { laboral }\end{array}$} & Desempregado & 32 & 14 \\
\hline & Emprego privado & 51 & 23 \\
\hline & Emprego público & 117 & 52 \\
\hline & Emprego sem vinculo & 20 & 9 \\
\hline & aposentado/reformado & 6 & 3 \\
\hline \multirow{5}{*}{$\begin{array}{l}\text { Atividade } \\
\text { voluntária }\end{array}$} & Nunca realizei & 50 & 22 \\
\hline & Realizei poucas vezes & 103 & 46 \\
\hline & Realizei uma vez & 34 & 15 \\
\hline & Realizo quase sempre & 30 & 13 \\
\hline & Realizo sempre & 9 & 4 \\
\hline \multirow{5}{*}{ Drogas } & Nunca utilizei & 75 & 33 \\
\hline & Utilizei algumas vezes & 85 & 38 \\
\hline & Utilizo quase sempre & 32 & 14 \\
\hline & Utilizo sempre & 19 & 8 \\
\hline & Utilizei uma vez & 15 & 7 \\
\hline \multirow{5}{*}{ Moradia } & Alugada e resido com familiares & 24 & 11 \\
\hline & Alugada e resido sozinho(a) & 10 & 4 \\
\hline & Outros & 4 & 2 \\
\hline & Própria e resido com familiares & 177 & 78 \\
\hline & Própria e resido sozinho(a) & 11 & 5 \\
\hline \multirow{5}{*}{ Alimentação } & $\begin{array}{l}\text { Alimento-me de tudo, sem } \\
\text { preocupação }\end{array}$ & 61 & 27 \\
\hline & $\begin{array}{l}\text { Dieta especifica (vegetariano, } \\
\text { vegano,etc.) }\end{array}$ & 3 & 1 \\
\hline & Saudável & 40 & 18 \\
\hline & Saudável, exceto algumas vezes & 120 & 53 \\
\hline & $\begin{array}{l}\text { Tenho alimentação básica em } \\
\text { fast-food }\end{array}$ & 2 & 1 \\
\hline
\end{tabular}

Tabela 2: continuação

\begin{tabular}{|c|c|c|c|}
\hline Variável & Categoria & Fi & $\%$ \\
\hline \multirow{5}{*}{ Lazer } & Não tenho dinheiro para & 16 & 7 \\
\hline & Não tenho tempo para & 22 & 10 \\
\hline & $\begin{array}{l}\text { Tenho dinheiro, mas não tenho } \\
\text { tempo }\end{array}$ & 36 & 16 \\
\hline & Tenho tempo e dinheiro para & 117 & 52 \\
\hline & $\begin{array}{l}\text { Tenho tempo, mas não tenho } \\
\text { dinheiro }\end{array}$ & 35 & 15 \\
\hline \multirow{5}{*}{$\begin{array}{l}\text { Atividade } \\
\text { física }\end{array}$} & Não realizo, por não gostar & 26 & 12 \\
\hline & Não realizo, por não ter dinheiro & 8 & 4 \\
\hline & Não realizo, por não ter tempo & 53 & 23 \\
\hline & Realizo esporádicamente & 60 & 27 \\
\hline & Realizo regularmente & 79 & 35 \\
\hline \multirow{4}{*}{ Amigos } & Acho que tenho alguns amigos & 21 & 9 \\
\hline & Não tenho muitos amigos & 11 & 5 \\
\hline & Tenho muitos amigos & 53 & 23 \\
\hline & $\begin{array}{l}\text { Tenho poucos, mas verdadeiros } \\
\text { amigos }\end{array}$ & 141 & 62 \\
\hline \multicolumn{2}{|l|}{ Total } & 266 & 100 \\
\hline
\end{tabular}

Fonte: dados da pesquisa dos autores.

Analisando as distribuições das 15 primeiras questões do instrumento, observe-se que "Gosta da família?" (A5), foi a questão com maior proporção de resposta "sempre", com $74 \%$. Por outro lado, "Sente-se sem esperança perante o futuro?" (A14), foi a questão com maior proporção de resposta "pouco tempo", com $40 \%$. Apresenta-se no quadro 1 os itens das primeiras questões do instrumento.

Quadro 1: Questões formuladas aos participantes da pesquisa

\begin{tabular}{l} 
A1. Sente-se bem consigo mesmo? \\
\hline A2. Tem se sentido uma pessoa feliz? \\
\hline A3. Sente-se bem na companhia das outras pessoas? \\
A4. Quando tem problemas que lhe incomodam, pode contar \\
com um ou mais amigos que lhe servem de confidentes? \\
\hline A5. Gosta da sua família? \\
\hline A6. O que você faz é valorizado pelos outros? \\
\hline A7. Sente-se bem com o seu corpo? \\
\hline A8. Habitualmente tem um sono reparador? \\
\hline A9. Gosta do que faz? \\
\hline A10. Considera-se satisfeito com o seu desempenho? \\
\hline $\begin{array}{l}\text { A11. Costuma dispor de dinheiro suficiente para satisfazer as } \\
\text { suas necessidades pessoais? }\end{array}$ \\
A12. É um tipo de pessoa capaz de rir dos acontecimentos \\
desagradáveis que lhe ocorrem? \\
A13. Você é uma pessoa determinada na resolução dos seus \\
problemas?
\end{tabular}

A14. Sente-se sem esperança perante o futuro?

A15. Mudaria alguma coisa na sua vida?

Fonte: dados da pesquisa dos autores. 
Em relação às demais questões, observou-se que "Fica nervoso e aborrecido quando não se sai tão bem quanto esperava ao realizar as suas tarefas?" (B3), foi a questão com maior proporção de resposta "todo o tempo", com $11 \%$. Por outro lado, "Tem pensamentos de acabar com a vida?" (B20), foi a questão com a maior proporção de resposta "nunca", com $85 \%$. Apresenta-se no Quadro 2 as questões da segunda parte do instrumento.

Quadro 2: Questões formuladas aos participantes da pesquisa

B1. Preocupa-se facilmente com os contratempos do dia-a-dia?

B2. Perante as dificuldades do dia-a-dia, você é mais para se queixar do que para se esforçar para resolvê-las?

B3. Fica nervoso e aborrecido quando não se sai tão bem quanto esperava ao realizar as suas tarefas?

B4. Fica nervoso e aborrecido se não obtém de forma imediata aquilo que quer?

B5. Você é uma pessoa que se irrita com facilidade?

B6. Tem impulsos que não consegue controlar?

B7. Tem impulsos de bater, ofender ou ferir alguém?

B8. Tem ataques de terror ou de pânico?

B9. Entra facilmente em discussão?

B10. Sente-se nervosa(o) quando tem que ficar sozinho(a)?

B11. Sente-se sozinho mesmo quando está com mais pessoas?

B12. Sente necessidade de evitar certas coisas, lugares ou atividades por the causarem medo?

B13. Costuma ter um medo súbito sem razão para isso?

B14. As pessoas só the dão atenção quando precisam que você faça alguma coisa em seu proveito?

B15. Sente que as outras pessoas não dão o devido valor ao seu trabalho ou às suas capacidades?

B16. Dedica mais tempo às solicitações das outras pessoas do que ás suas próprias necessidades?

B17. Prefere calar-se, do que contrariar alguém, mesmo que não tenha razão?

B18. Tem impressão de que outras pessoas podem controlar os seus pensamentos?

B19. Sente-se inferior aos outros?

B20. Tem pensamentos de acabar com a vida?

B21. Possui sentimentos de culpa?

B22. Tem a ideia que deveria ser castigado pelos seus pecados?

B23. Em determinadas situações, tem dificuldade em exprimir aquilo que sente?

B24. Tem dificuldade em tomar decisões?

B25. Tem dificuldades em se concentrar?

B26. Sente necessidade de verificar várias vezes o que faz?

B27. Tem dificuldades em adormecer?

B28- Acorda demasiadamente cedo sem querer?

B29. Tem a sensação de que the falta o ar?

B30. Acredita que na maior parte dos casos as soluções para os problemas importantes da sua vida não dependem de você?

B31. Considera que os outros são culpados pela maioria dos seus problemas?

Fonte: dados da pesquisa dos autores.

Observação: Itens do AGNSM - segunda parte
Antes de dar início ao cálculo do Coeficiente de Correlação de Spearman, da Análise Fatorial e do cálculo de Alpha de Cronbach, foi necessário redefinir algumas codificações, de forma que todas as questões variassem em uma escala Likert de 1 a 5, onde 1 é a menor pontuação e 5 a maior pontuação. Essas codificações foram definidas da seguinte forma: Pouco tempo: 1; Algum tempo: 2; Bastante tempo: 3; Quase sempre: 4; Sempre: 5. Nunca: 1; Pequena parte do tempo: 2; Uma boa parte do tempo: 3; A maior parte do tempo: 4; Todo o tempo: 5 .

Em seguida, foi avaliado via Coeficiente de Spearman, a correlação entre todas as 46 questões do instrumento. A Tabela 3 apresenta todos os coeficientes calculados, seguido do $p$-valor sob a hipótese de que esse coeficiente é igual a zero. Dessa forma, rejeitar essa hipótese ( $p$-valor menor que $0,05)$, significa que a correlação calculada é estatisticamente diferente de zero. Vale salientar que foram identificadas dezenas de correlações estatisticamente significativas.

Para uma melhor apresentação dos resultados, as questões do instrumento foram renomeadas, sendo as 15 primeiras questões nomeadas de $\mathrm{A} 1$ até $\mathrm{A} 15$, e as últimas 31 questões nomeadas de B1 até B31. Antes de seguir com o desenvolvimento da Análise Fatorial, foi calculado o Alpha de Cronbach geral do instrumento, considerando todas as 46 questões abordadas, e o valor obtido foi de 0,871, que é um valor alto, conforme Hora, Monteiro e Arica, (2010), indicando que o instrumento produz mensurações estáveis e consistentes. Foi calculado ainda o Alpha de Cronbach separado para o primeiro bloco de questões (primeiras 15) e para o último bloco (últimas 31), e os valores obtidos foram de 0,810 e 0,945, respectivamente, indicando que essas questões, mesmo em blocos separados, são capazes de produzir mensurações estáveis e consistentes. ${ }^{5}$

A Análise Fatorial foi desenvolvida para todas as 46 questões, onde observou-se significância estatística do Teste de Bartlett (p-valor menor que 0,0001). 0 teste de Bartlett permite confirmar a possibilidade e adequação do método de Análise Fatorial para o tratamento dos dados ao verificar se há correlações desejáveis entre as variáveis. ${ }^{6} \mathrm{Um}$ teste significativo, que é o caso desse estudo, mostra que a matriz de correlações não é uma matriz de identidade, e que, portanto, há algumas relações entre as variáveis que se espera incluir na análise, justificando a aplicação da Análise Fatorial. Esse teste é sensível ao tamanho da amostra e por isso convém usar também o MSA de KaiserMeyer-Olkin (KMO).

A medida de KMO varia entre zero e um e compara as correlações simples com as correlações parciais observadas entre as variáveis. Um resultado maior que 0,5 indica que a análise fatorial é adequada para a amostra utilizada. 0 resultado obtido aqui foi de 0,9050, o que é admirável. Esses scores corroboram com os resultados, onde foram identificadas dezenas de correlações estatisticamente significativas entre as questões.

Pela análise fatorial, foi possível extrair 10 fatores com carga fatorial acima de 0,40 , sendo que a quantidade de 
fatores é definida pela quantidade de autovalores maiores que $1^{(6)}$, explicando assim $65 \%$ da variabilidade dos dados. No primeiro fator a questão B8 foi a que apresentou maior carga fatorial, o que indica que essa é a questão com maior peso na definição desse fator. No fator 2, a questão com maior carga foi a A10 - Considera-se satisfeito com o seu desempenho? e no fator 3 foi a B4-Fica nervoso e aborrecido se não obtém de forma imediata aquilo que quer?; no fator 4 foi a B27. Tem dificuldades em adormecer?; no fator 5 foi a B24. Tem dificuldade em tomar decisões?, no fator 6 foi a B17 - Prefere calar-se, do que contrariar alguém, mesmo que não tenha razão?, no fator 7 foi a B30. Acredita que na maior parte dos casos as soluções para os problemas importantes da sua vida não dependem de você? e no fator 8 foi a A14. Sente-se sem esperança perante o futuro?. Os fatores 9 e 10 foram compostos, exclusivamente, pelas questões A4. Quando tem problemas que lhe incomodam, pode contar com um ou mais amigos que the servem de confidentes? e A5. Gosta da sua família?, respectivamente. A questão B25 - Tem dificuldades em se concentrar? não apresentou carga fatorial maior que 0,4 em nenhum dos fatores selecionados (autovalor maior que 1).

Feito isso, calculou-se o Alpha de Cronbach para o grupo de questões que compõem cada um dos fatores obtidos na Análise Fatorial. Os fatores 1 a 5 apresentaram valores de Alpha de Cronbach altos, sendo 0,9177, 0,8747, 0,8388, 0,7190 e 0,7525 , respectivamente, bem como o fator 7 , com 0,7356. Todos esses fatores produzem, separadamente, mensurações estáveis e consistentes. Os únicos fatores que apresentaram Alpha de Cronbach abaixo de 0,6 foram o fator 6 e 8, com 0,5729 e 0,5269, respectivamente, indicando que esses fatores, especificamente, podem estar produzindo mensurações instáveis e inconsistentes.

Tabela 3. Comparação das características da amostra com o escore total e os domínios da Escala de Avaliação da Satisfação com a Imagem Corporal. São Paulo, 2014-2015.

\begin{tabular}{|c|c|c|c|c|c|c|c|c|c|c|c|}
\hline \multirow{2}{*}{ Fator } & \multirow{2}{*}{ Questão } & \multicolumn{10}{|c|}{ Cargas Fatoriais } \\
\hline & & Fator 1 & Fator 2 & Fator 3 & Fator 4 & Fator 5 & Fator 6 & Fator 7 & Fator 8 & Fator 9 & Fator 10 \\
\hline \multirow{13}{*}{ Fator 1} & B6 & 0.5780 & & & & & & & & & \\
\hline & B7 & 0.6758 & & & & & & & & & \\
\hline & B8 & 0.8247 & & & & & & & & & \\
\hline & B9 & 0.524 & & & & & & & & & \\
\hline & B10 & 0.6371 & & & & & & & & & \\
\hline & B11 & 0.5647 & & & & & & & & & \\
\hline & B12 & 0.6764 & & & & & & & & & \\
\hline & B13 & 0.7928 & & & & & & & & & \\
\hline & B18 & 0.5892 & & & & & & & & & \\
\hline & B20 & 0.6070 & & & & & & & & & \\
\hline & B21 & 0.4572 & & & & & & & & & \\
\hline & B22 & 0.4995 & & & & & & & & & \\
\hline & B29 & 0.5668 & & & & & & & & & \\
\hline \multirow{11}{*}{ Fator 2} & $\mathrm{~A} 1$ & & 0.7422 & & & & & & & & \\
\hline & $\mathrm{A} 2$ & & 0.6320 & & & & & & & & \\
\hline & A3 & & 0.6387 & & & & & & & & \\
\hline & A6 & & 0.5641 & & & & & & & & \\
\hline & A7 & & 0.6141 & & & & & & & & \\
\hline & A8 & & 0.4619 & & & & & & & & \\
\hline & A9 & & 0.7731 & & & & & & & & \\
\hline & $\mathrm{A} 10$ & & 0.7993 & & & & & & & & \\
\hline & A11 & & 0.5382 & & & & & & & & \\
\hline & A12 & & 0.4508 & & & & & & & & \\
\hline & A13 & & 0.6581 & & & & & & & & \\
\hline \multirow{5}{*}{ Fator 3} & B1 & & & 0.5267 & & & & & & & \\
\hline & B3 & & & 0.6713 & & & & & & & \\
\hline & B4 & & & 0.7707 & & & & & & & \\
\hline & B5 & & & 0.7354 & & & & & & & \\
\hline & B26 & & & 0.4787 & & & & & & & \\
\hline
\end{tabular}


Tabela 3. Continuação

\begin{tabular}{|c|c|c|c|c|c|c|c|c|c|c|c|}
\hline \multirow{2}{*}{ Fator } & \multirow{2}{*}{ Questão } & \multicolumn{10}{|c|}{ Cargas Fatoriais } \\
\hline & & Fator 1 & Fator 2 & Fator 3 & Fator 4 & Fator 5 & Fator 6 & Fator 7 & Fator 8 & Fator 9 & Fator 10 \\
\hline \multirow{2}{*}{ Fator 4} & B27 & & & & 0.7416 & & & & & & \\
\hline & B28 & & & & 0.7193 & & & & & & \\
\hline \multirow{3}{*}{ Fator 5} & B19 & & & & & 0.4852 & & & & & \\
\hline & $\mathrm{B} 23$ & & & & & 0.5499 & & & & & \\
\hline & B24 & & & & & 0.6936 & & & & & \\
\hline \multirow{2}{*}{ Fator 6} & B16 & & & & & & 0.6443 & & & & \\
\hline & B17 & & & & & & 0.7100 & & & & \\
\hline \multirow{4}{*}{ Fator 7} & B14 & & & & & & & 0.5018 & & & \\
\hline & B15 & & & & & & & 0.4508 & & & \\
\hline & B30 & & & & & & & 0.6947 & & & \\
\hline & B31 & & & & & & & 0.5124 & & & \\
\hline \multirow{3}{*}{ Fator 8} & A14 & & & & & & & & 0.6527 & & \\
\hline & A15 & & & & & & & & 0.6477 & & \\
\hline & B2 & & & & & & & & 0.5275 & & \\
\hline Fator 9 & A5 & & & & & & & & & 0.7728 & \\
\hline \multirow[t]{2}{*}{ Fator 10} & A4 & & & & & & & & & & 0.5993 \\
\hline & B25 & & & & & & & & & & \\
\hline Autovalor & & 13.82 & 5.03 & 1.74 & 1.67 & 1.58 & 1.47 & 1.24 & 1.10 & 1.08 & 1.02 \\
\hline $\begin{array}{l}\% \text { da } \\
\text { variabilidade }\end{array}$ & & $30 \%$ & $11 \%$ & $4 \%$ & $4 \%$ & $3 \%$ & $3 \%$ & $3 \%$ & $2 \%$ & $2 \%$ & $2 \%$ \\
\hline $\begin{array}{l}\% \text { acumulado } \\
\text { da variabilidade }\end{array}$ & & $30 \%$ & $41 \%$ & $45 \%$ & $48 \%$ & $52 \%$ & $55 \%$ & $58 \%$ & $60 \%$ & $62 \%$ & $65 \%$ \\
\hline $\begin{array}{l}\text { Alpha de } \\
\text { cronbach }\end{array}$ & & 0.9177 & 0.8747 & 0.8388 & 0.7190 & 0.7525 & 0.5729 & 0.7356 & 0.5269 & - & - \\
\hline $\begin{array}{l}\text { Bartlett } \\
\text { (p-valor) }\end{array}$ & & & & & & & & & & & $<.0001$ \\
\hline $\mathrm{KMO}^{*}$ & & & & & & & & & & & \\
\hline
\end{tabular}

Fonte: dados da pesquisa dos autores.

KMO* - Medida de Kaiser-Meyer-Olkin.

\section{DISCUSSÃO}

Os resultados deste estudo demonstram a forte confiabilidade e validade do construto da escala de Avaliação Global do Nível de Saúde Mental (AGNSM), em uma população de 266 respondentes de três países: Brasil, Espanha e Portugal, confirmando assim o modelo teórico proposto. Em relação à confiabilidade da escala, os resultados mostram que há boa consistência interna e homogeneidade dos itens. Especificamente, essa escala obteve um alto nível de confiabilidade com o Alfa de Cronbach com valores maiores do que 0,80 na maioria das dimensões.

Ressalta-se que os únicos fatores que apresentaram Alpha de Cronbach abaixo de 0,6 foram o fator 6 e 8, com 0,5729 e 0,5269, respectivamente, indicando que esses fatores, especificamente, podem estar produzindo mensurações instáveis e inconsistentes, mesmo assim, sua baixa variabilidade dentro da escala não afetou significativamente os bons níveis de confiabilidade.
As medidas estatísticas para validar o construto em cada uma das três dimensões originalmente propostas apontam boa relação entre os itens. Essas análises também mostram a contribuição de cada item para a sua respectiva subescala, confirmando a pertinência das variáveis ou itens propostos. Do mesmo modo, a análise fatorial contribui para a validação do construto ao demonstrar que os itens tendem a se agrupar nas dimensões propostas pelos autores da escala.

\section{CONCLUSÃO}

A tridimensionalidade e a confiabilidade dessa escala são confirmadas pelos resultados, que asseguram a qualidade do instrumento na população estudada. Com base nos dados apresentados, é possível estabelecer que a aplicação da escala AGNSM, avalia o nível global de saúde mental. Além disso, essa escala tem características, como sua facilidade de aplicação, que permitem coletar 
respostas de um grande número de indivíduos. Assim, a escala representa uma maneira fácil e econômica de medir o construto proposto.

Vale evidenciar que se trata de um instrumento que avalia o nível de saúde mental, ou seja, num continuo integrando os fatores de vulnerabilidade e de proteção, algo que não existe nos instrumentos disponíveis localizados na literatura, tendo em vista que observouse na busca realizada, que os instrumentos existentes se focam essencialmente em um dos polos, permitindo avaliar apenas um dos aspectos de cada vez, positivos ou negativos, o que compõe um forte diferencial do AGNSM a possibilidade de avaliar os diversos fatores.

Após analisadas as propriedades psicométricas do AGNSM, verifica-se que este apresenta boa fidelidade e validade de constructo. Neste sentido, pode-se considerálo um instrumento útil na avaliação das fontes e intensidade de estresse nos diversos grupos humanos, uma vez que a literatura nos chama a atenção para a necessidade de considerar as diferenças culturais, o que permitiu comparativos dos fatores, níveis e respostas dos sujeitos pesquisados, no que tange à saúde mental. Essa característica reflete a forte validade transcultural do instrumento de medida AGNSM.

Face ao resultado deste, salienta-se a importância de serem desenvolvidos estudos que possibilitem a análise fatorial confirmatória, de forma a avaliar a fidelidade deste instrumento, bem como o estabelecimento de pontos de corte, que permitam identificar as pessoas em maior risco ou maior vulnerabilidade.

\section{REFERÊNCIAS}

1. Sequeira CAC, Carvalho JC, Sampaio F, Sá L, Canut MTL, Merino JR. Avaliação das propriedades psicométricas do questionário de saúde mental positiva em estudantes portugueses do ensino superior. Revista Portuguesa de Enfermagem de Saúde Mental. 2014;(11):45-53.

2. Sequeira C, Carvalho JC. Tradução para a População Portuguesa do Questionário de Saúde Mental Positiva (QSM+). In: Sequeira CA, Carvalho JC, Ribeiro I, Martins T, Rodrigues T. Saúde e Qualidade de Vida em análise. Porto (PT): ESEP; 2009. p. 303-314.

3. Cannut MTL. Concepto de salud mental positiva: Factores relacionados. In Fornes J, Gómez J. Recursos y programas para la salud mental. Enfermería psicosocial II. Madrid (ES):

4. Fuden, Colección líneas de especialización en enfermería. 2008. p. 37-69.

5. Canut MTL, Llobet MP, Ortega AS, Merino JR, Grau CF. \& Positive Mental Health Research Group. Assessing positive mental health in people with chronic physical health problems: correlations with socio-demographic variables and physical health status. BMC Public Health. 2013; (13): 9-28.

6. Hora HRM, Monteiro GTR, Arica J. Um Estudo com o Coeficiente Alfa de Cronbach. Produto \& Produção [Internet]. 2010. [Acessado em 28 maio 2015];11(2):85-103. Disponível em: http://seer.ufrgs.br/index.php/ProdutoProducao/article/ viewFile/9321/8252.

7. Venegas ME, Alvarado OS, Elizondo NR, Carrillo KS. Validação do construto e da confiabilidade de uma escala de inteligência emocional aplicada a estudantes de enfermagem. Rev. Latino-Am Enferm [Internet]. 2015. [Acessado em 15 de junho 2015];23(1):139-147. DOI: 10.1590/0104-1169.3498.2535. Disponível em: http://www.scielo.br/pdf/rlae/v23n1/ pt_0104-1169-rlae-23-01-00139.pdf.

8. Bakker AB, Demerouti E, Euwema M.Job Reaources Buffer The Impact of Job Demands on Burnout. Journal of Occupational Health Psychology. 2005; 10(2): 170-180.

9. Bakker AB, Van Der Zee K, Lewing K, Dollard M. (2006). The Relationship Between the Big Five Personality Factors and Burnout: A Study Among Volunteer Counselors. The Journal of Social Psychology. 2006;146(1): 31-50.

10. Bishop G, Enkelmann H, Tong E, Why Y, Diong S, Khader M, Ang J. Job Demands, Decisional Control, and Cardiovascular Responses. Journal of Occupational Health Psychology. 2003; 8(2):146-156.

11. Cooper G, Marshall J. Fontes Ocupacionais de "Stress": Uma revisão da literatura relacionada com Doenças Coronárias e com Saúde Mental. Análise Psicológica. 1982;11(2/3): 153-170.

12. Folkman S. Personal Control and Stress and Coping Processes: A theoretical Analysis. Journal of Personality and Social Psychologi. 1984;46(4):838-52.

13. Monte PG, Peiró JA. Model of Burnout Process Development:an Alternative from Appraisal Model of Stress. Comportamento Organizacional e Gestão. 1998;4(1):165-79.

14. Hart P. Predicting Employee Life Satisfaction: A Coherent Model of Personality, Work and Nonwork Experiences, and Domain Satisfactions. Journal of Applied Psychology. 1999;84(4): 564-584.

15. Hilouse J, Adler CM. Evaluating a simple Model of Work Stress, Burnout, Affective and Physical Simptoms in Hospital Nurses. Psychology, Health \& Medicine. 1996;10(3):297-306.

16. Maslach C.A Multidimensional Theory of Burnout. In:Theories of Organizational Stress. Cooper GL. Oxford University Press. New York: (USA). 1998. p. 68-85. 